Trauma Berufskrankh 2008 10 [Suppl 3]:327-330

DOI 10.1007/s10039-007-1345-x Online publiziert: 6. März 2008

(c) Springer Medizin Verlag 2008

A. Rahmanian-Schwarz $\cdot$ M. Pfau $\cdot$ H.E. Schaller

Klinik für Hand-, Plastische, Rekonstruktive und Verbrennungschirurgie,

BG-Unfallklinik, Tübingen

\title{
Nachsorge bei Verbrennung
}

Seit den ersten Therapieansätzen zur Oberflächenbehandlung von Brandwunden durch tierische Fette aus dem Jahre 2900 v. Chr. hat sich die Verbrennungsmedizin stark differenziert. Während bis vor kurzem das Überleben des schwer verbrannten Patienten maßgeblich für das Therapiemanagement war, ist heute die Reintegration im privaten, beruflichen und gesellschaftlichen Leben das übergeordnete Ziel. Mittlerweile bieten spezielle Verbrennungszentren ganzheitliche, interdisziplinäre Behandlungskonzepte auf höchstem Niveau.

Die Prognose und der Verlauf nach Verbrennungen hängen von vielen Faktoren ab wie

- Lokalisation,

- Tiefengrad,

- Ursache der Verbrennung und

- Alter des Patienten.

Im Vergleich zu anderen Verletzungen sind die thermischen Schäden durch eine langwierige Therapie und dauerhafte Folgen gekennzeichnet. In einer Studie von Zeitlin et al. [11] wurde berichtet, dass bei kindlichen Verbrühungen ab einer Größe von $5 \%$ der Körperoberfläche in $95 \%$ der Fälle nach 17 Jahren noch eine sichtbare Narbe verbleibt.

Die moderne Verbrennungstherapie ist in 4 Hauptphasen der Patientenversorgung gegliedert:

Phase 1. Im Zeitraum vom 1.-3. Tag nach der Verbrennung erfolgt die Akutbehandlung. Dabei geht es hauptsächlich um die Überlebenssicherung des Verbrannten mit Evaluation des Verbrennungsausmaßes und der Begleitverletzungen. Darüber hinaus ist die Flüssigkeitssubstituti- on ein wesentlicher Bestandteil der Akutphasentherapie.

Phase 2. Sie beinhaltet die initiale Wundexzision mit temporärer Deckung, die stufenweise innerhalb der ersten Tage nach der Akutversorgung erfolgt.

Phase 3. In ihr werden der definitive Wundverschluss durch Hauttransplantation und die akute Rekonstruktion der komplexen Körperregionen wie Gesicht und Hände durchgeführt.

Phase 4. Die Nachsorge ist durch eine langfristige Rehabilitation, Rekonstruktion und Reintegration des verbrannten $\mathrm{Pa}$ tienten gekennzeichnet.

Die sichtbaren Entstellungen und Kontrakturen führen häufig zum Verlust der Selbstständigkeit und zur Isolation der Brandopfer. Diese seelischen und funktionellen Einschränkungen verlangen ein umfassendes Rehabilitationsprogramm mit interdisziplinärer Teamarbeit sowie einem individuell angepassten Therapiekonzept unter Einbeziehung des sozialen Umfelds. Die notwendigen Voraussetzungen an Fachkompetenz, Erfahrung und medizinischer Ausstattung für eine langfristige Planung und Durchführung der Therapie sind speziellen Verbrennungszentren vorbehalten. Diese sind in der Lage, die hohen Anforderungen an medizinisch-funktionelle Behandlungen zu erfüllen und den Betroffenen individuell psychologisch zu betreuen. Weiterhin haben sie die Möglichkeit, im Rahmen der Nachsorge dem Patienten ein umfassendes soziales und berufliches Reintegrationsprogramm anzubieten.

\section{Nachsorge des Verbrannten}

Neben der Akutbehandlung mit Überlebenssicherung des schwer verbrannten Patienten stellt die Nachsorge eine besondere Herausforderung an alle an der Therapie beteiligten Personen dar. Ein umfassendes Nachsorgeprogramm setzt sich aus Rehabilitation, Rekonstruktion und Reintegration des Betroffenen zusammen. Es sollte frühzeitig im multidisziplinären Team geplant werden und individuell angepasste, operative und konservative Maßnahmen in die Therapie einbinden. In der Regel besteht das interdisziplinäre Regime aus:

- psychologischen,

- physiotherapeutischen,

- sozialmedizinischen,

- pflegerischen und

- operativen Einheiten.

Bei der Zielsetzung sollten Faktoren wie Ausmaß der Verletzung, Patientenalter, Komorbidität, berufliche Exposition und private Verhältnisse des Verletzten berücksichtigt werden. Des Weiteren ist eine realistische Planung, unter Beteiligung aller Behandlungsteams und Angehörigen, entscheidend für den langfristigen Therapieerfolg. Dabei sollten sich die primären Ziele zunächst an den Grundbedürfnissen des Patienten wie Stehen, Gehen und Essen orientieren. Auch ein adäquates Zeitmanagement spielt hinsichtlich der Dauer und des Beginns der Therapieeinheiten eine wesentliche Rolle in der Nachsorge. Im Rahmen eines Therapeutenassessments sollten der Verlauf der Behandlung und die Ergebnisse regelmäßig diskutiert und evaluiert werden. 


\section{Verbrennungstherapie}
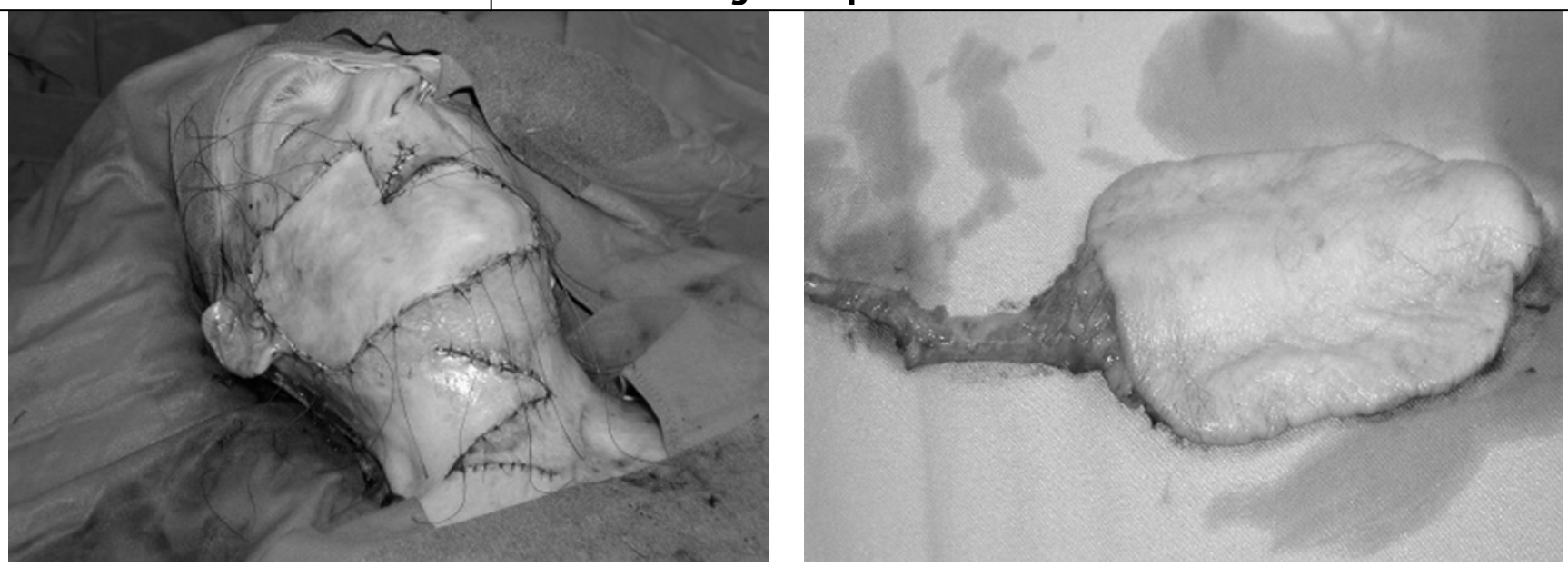

Abb. $1 \Delta$ Versorgung durch Vollhauttransplantation, Z-Plastik

Abb. 2 \ Versorgung durch Radialislappen
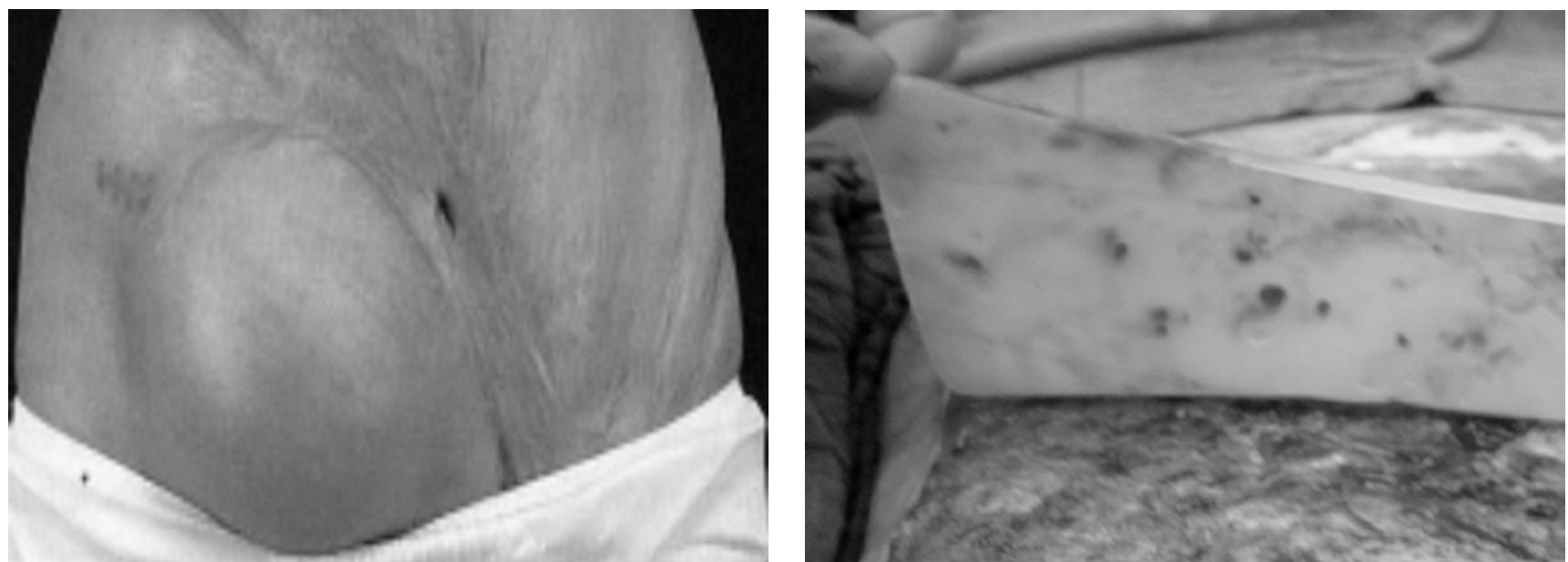

Abb. $3 \Delta$ Expandereinsatz

Abb. $4 \Delta$ Dermaler Ersatz (Integra ${ }^{\circledR}$ )

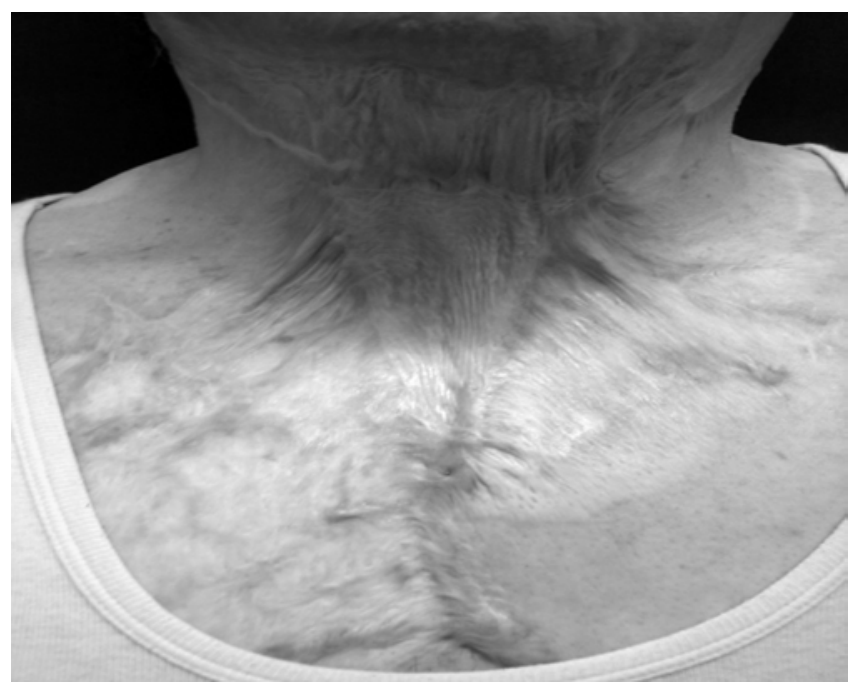

Abb. $5<$ Narbenkontraktur mentosternal
Schließlich kann nur ein professionelles Nachsorgekonzept unter gleichzeitiger Berücksichtigung der Rehabilitation, Rekonstruktion und Reintegration des Patienten langfristig zum Erfolg führen.

\section{Rehabilitation}

\section{Akutphase}

Bereits in der Frühphase der Behandlung ist die Rehabilitation des Verbrannten durch erfahrene Therapeuten für den Funktionserhalt entscheidend. Eine intensive physiotherapeutische Betreuung, mehrmals täglich, mit funktioneller Beübung der Extremitäten, sollte begleitend in der Akutphase eingeleitet werden. Dabei ist es das primäre Ziel, die passiven ROM („rang of motion“) an den Extremitäten zu erhalten. Zur Kontrakturprophylaxe ist eine individuelle Schienenbehand- 
lung mit regelmäßiger Anpassung indiziert. Diese Maßnahmen lassen sich besonders gut in der Akutphase durchführen, da die Patienten dann noch sediert und beatmet sind.

Außerdem ist die Einbeziehung der Angehörigen bereits zu Beginn der Behandlung entscheidend, da sie besonders bei der Langzeittherapie des Verbrannten eine wesentliche Rolle übernehmen und die Compliance positiv beeinflussen können.

\section{Heilungsphase}

Hat der Patient die akute Therapiephase überstanden, werden die Rehabilitationsmaßnahmen erweitert. Es kommt zur Steigerung der passiven Mobilisation bis hin zu aktiven Bewegungsübungen und Kraftaufbau. Neben der gesteigerten Bewegungstherapie wird ein an die Bedürfnisse angepasstes ergotherapeutisches Konzept erstellt. Im Vergleich zur Frühphase ist der Umgang mit den nun wachen Patienten mit zunehmenden posttraumatischen Belastungssymptomen schwierig und zeitintensiv.

Die Zusammenarbeit und Koordination zwischen verschiedenen Therapeutenteams haben sich besonders in dieser Phase bewährt. So können beispielsweise die Mobilisation und die passiven Bewegungsübungen durch Krankengymnasten im Rahmen des chirurgischen Verbandswechsels und der Wundkontrolle die Sedierung und den Analgesiebedarf des Patienten verringern.

\section{Rekonstruktion}

Auch wenn eine adäquate Akutbehandlung das Ausmaß an rekonstruktiven Eingriffen verringert, bedarf es im Verlauf des ersten Behandlungsjahrs einiger Korrekturoperationen. Sie sollten jedoch erst nach ausreichender Wundheilung und -stabilisierung erfolgen und primär die fehlende Funktionalität wiederherstellen. Ein verfrühter Eingriff kann zur Unterbrechung der Reintegration und einem verlängerten Heilungsprozess führen. Ausnahmen sind Eingriffe im KopfHals-Bereich und an den Händen, wo es einer frühzeitigen, funktionellen Rekonstruktion bedarf. Die Wiederherstellung der betroffenen Areale kann je nach Er- fordernis durch unterschiedliche chirurgische Techniken erfolgen:

- Inzision und Exzision mit freier Hauttransplantation (• Abb. 1)

- Z-Plastiken und lokale Verschiebeplastiken (• Abb. 1)

- Gestielte lokale Nah- und Fernlappen

- Freie mikrovasluläre Lappen (- Abb. 2)

- Gewebeexpander (• Abb. 3)

- Dermaler Ersatz (Integra ${ }^{\circ}$, Matriderm', usw., D Abb.4)

\section{Narbenmanagement}

Eine der Hauptkomplikationen in der Nachsorge verbrannter Patienten sind die Naben und Narbenkontrakturen. Definitionsgemäß sind Narben vollständig epithelialisierte, dermale Läsionen, die über das Niveau der intakten Haut ragend in unterschiedlicher Form, Farbe und Konsistenz vorkommen ( $\mathbf{\bullet}$ Abb. 5). Sie bilden sich in der Regel 3 Wochen bis 6 Monate nach der thermischen Schädigung.

Die Therapie der hypertrophen Narbe ist ein essenzieller Aspekt in der Nachsorge des verbrannten Patienten. Besonders betroffen sind Körperregionen mit hoher elastischer Eigenschaft, wie Gesicht, Hals und Hände.

Die Behandlungsoptionen sind sowohl in der Anzahl als auch in ihrer Effizienz begrenzt. Sie gestalten sich operativ und konservativ. Narbenmassage, angepasste Kompressionskleider und Silikonauflagen gehören zu den konservativen Maßnahmen, die bei einer regelmäßigen und intensiven Anwendung mit guten Ergebnissen einhergehen können. Bei der Kortisontherapie handelt es sich um eine lokal begrenzte Injektion, die häufig mit starken Schmerzen verbunden ist.

Chirurgisch lassen sich die Narben durch Inzision, Exzision, Lappenplastiken in unterschiedlicher Form und Hauttransplantationen korrigieren. Der Zeitpunkt der Rekonstruktion ist entscheidend, denn jede Operation im Rahmen der Nachsorge führt zur Unterbrechung der Reintegration und kann den Heilungsverlauf verlängern.
Trauma Berufskrankh 2008 10

[Suppl 3]:327-330

DOI 10.1007/s10039-007-1345-x

○) Springer Medizin Verlag 2008

A. Rahmanian-Schwarz - M. Pfau • H.E. Schaller

Nachsorge bei Verbrennung

\section{Zusammenfassung}

Während früher das Überleben des schwerverbrannten Patienten maßgeblich für das Therapiemanagement war, steht heute dessen Reintegration im Alltag im Vordergrund. Die moderne Verbrennungstherapie ist in 4 Hauptphasen gegliedert: Phase 1: Akutbehandlung, Phase 2: Wundexzision mit temporärer oder definitiver Deckung, Phase 3: akute Rekonstruktion und definitiver Wundverschluss sowie Phase 4: Nachsorge. Letztere beinhaltet die Rehabilitation, Rekonstruktion und Reintegration und wird im vorligenden Beitrag detailliert besprochen.

\section{Schlüsselwörter}

Moderne Verbrennungstherapie - Akutbehandlung · Rehabilitation - Rekonstruktion . Reintegration

\section{Aftercare for burns}

\section{Abstract}

Until recently, the survival of severely burned patients was of primary importance for therapy management. Today, reintegrating the patient into everyday life is at the forefront of treatment. Current burn therapy is divided into four main phases: phase 1: acute treatment; phase 2: wound excision with temporary covering; phase 3: wound closure and acute reconstruction; phase 4: aftercare, including rehabilitation, reconstruction and reintegration. The aftercare phase will be discussed in detail in the presented article.

\section{Keywords}

Current burn therapy - Acute treatment $\cdot$ Rehabilitation $\cdot$ Reconstruction $\cdot$ Reintegration 


\section{Reintegration und psychologische Aspekte}

Ein erfolgreicher Reintegrationsprozess sollte den Brandvorletzten bis zur Entlassung ausreichend auf seine Umwelt und seine Bedürfnisse vorbereiten. Poststationär leiden die Patienten häufig unter Anpassungsstörungen im Alltagsleben und müssen ihre Grenzen neu wahrnehmen.

In den ersten 18. Monaten poststationär zeigt sich oft eine Verschlechterung der Ergebnisse durch Therapeutenwechsel, mangelnde Verlaufkontrollen und Motivationsverlust des Betroffenen. Besonders in dieser Zeit sind die Einstellung und Psyche des Patienten ein wesentlicher Faktor für den langfristigen Therapieerfolg. Daher sollte die psychologische Betreuung des Verletztern bereits früh beginnen und ihn in allen Phasen der Behandlung begleiten. Posttraumatische Stressreaktionen können dennoch immer wieder durch Krisensituationen und Schlüsselreize ausgelöst werden. Die individuellen Coping-Strategien reichen oft nicht aus, die lebensbedrohlichen Ereignisse zu verarbeiten. Die Betroffenen versuchen, auslösende Faktoren zu meiden und flüchten in die Isolation. Ohne eine stabile Arzt-Patienten-Beziehung kommt es zu Depression und Demotivation. Dieses Verhalten überträgt sich unmittelbar auf alle am Behandlungsprozess Beteiligten und erfordert einen adäquaten Umgang und genaue Kenntnisse des Störungsbilds. Die psychologische Betreuung und ein geschulter Umgang mit den Brandverletzten sind daher ein wichtiger Bestandteil der Reintegration.

\section{Schlussfolgerung}

Die hohen Anforderungen an die Therapie des verbrannten Patienten können nur von speziellen Verbrennungszentren gewährleistet werden.

Eine realistische Zielsetzung mit Beteiligung aller Therapieteams und Angehörigen ist entscheidend für den langfristigen Therapieerfolg.

Das primäre Ziel der Rehabilitation in der Akutphase sind die passive Mobilisation und Kontrakturprophylaxe.

Der Zeitpunkt der Rekonstruktion ist entscheidend, denn jede Operation im Rah- men der Nachsorge führt zur Unterbrechung der Reintegration und kann den Heilungsverlauf verlängern.

Häufig leiden die Patienten unter einer posttraumatischen Belastungsstörung. Sie manifestiert sich in immer wiederkehrenden Erinnerungen an das Trauma (Flashbacks), die Depressionen, Angst oder Wut verursachen. Die psychologische Betreuung und ein geschulter Umgang mit den Brandverletzten sind daher ein wichtiger Bestandteil der Reintegration.

\section{Korrespondenzadresse}

Dr. A. Rahmanian-Schwarz

Klinik für Hand-, Plastische, Rekonstruktive und Verbrennungschirurgie BG-Unfallklinik Tübingen, Schnarrenbergstraße 95, 72076 Tübingen arahmanian@bgu-tuebingen.de

Interessenkonflikt. Der korrespondierende Autor gibt an, dass kein Interessenkonflikt besteht.

\section{Literatur}

1. Aikawa N, Wakabayashi G, Ueda M et al. (1990) Regulation of renal function in thermal injury. J Trauma [Suppl 12] 30: 174-178

2. Deitch EA, Winterton J, Li M et al. (1987) The gut is a portal of entry or bacteremia. Ann Surg 205: 681-690

3. Henckel von Donnersmarck G, Mühlbauer W (1996) Verbrennungen. Zuckschwerdt, München

4. Herndon DN (1996) Total burn care. Saunders, Philadelphia

5. Hettich R (1996) Die Grob'sche Gerbung (modifiziert nach Hettich). In: Krupp S (Hrsg) Plastische Chirurgie, Klinik und Praxis. Ecomed, Landsberg

6. Lönnecker S (1995) Die Erstversorgung des schwerbrandverletzten Patienten aus anästhesiologischer Sicht. Unfallchirurgie 98: 184-186

7. Marino PL (1994) Das ICU Buch, Praktische Intensivmedizin. Urban \& Schwarzenberg, München Wien Baltimore

8. Mühlbauer W, Olbrisch RR, Herndl E (1981) Treatment of neck contracture after burns with a free underarm flap. Chirurg 52: 635-637

9. Muller MJ (1994) The challenge of burns. Lancet 343: $216-220$

10. Rousselot LM, Connell JF, Whalen WP et al. (1953) The exposure method in the treatment of severe second and third degree burns. Surgery 33: 673682

11. Zeitlin R, Järnberg J, Somppi E et al. (1997) The late appearance of scars after burns in childhood. Scand J Plast Reconstr Surg Hand Surg 31: 319325

12. Zellweger G (1985) Die Behandlung der Verbrennungen, 2. Aufl. Deutscher Ärzteverlag, Köln 